\title{
Rate dependence of the magnetocaloric effect in La-Fe-Si compounds
}

\author{
M.Kuepferling ${ }^{1}$, C.P.Sasso ${ }^{1}$ and V.Basso ${ }^{1}$ \\ ${ }^{1}$ Istituto Nazionale di Ricerca Metrologica (INRiM), Division Electromagnetism, I-10135 Torino, Italy
}

\begin{abstract}
The dynamic magnetocaloric effect in $\mathrm{La}\left(\mathrm{Fe}_{\mathrm{x}} \mathrm{Co}_{\mathrm{y}} \mathrm{Si}_{1-\mathrm{x}-\mathrm{y}}\right)_{13}$ with $x=0.9$ and low $\mathrm{Co}$ content of $y=0.015$ was analysed by calorimetric measurements at constant magnetic field and constant temperature as well as magnetisation relaxation measurements. It is shown that the rate dependence of the measurement, which leads to an increased entropy hysteresis with increasing rate of the driving force (temperature or magnetic field), can be mainly attributed to a thermal contact resistance $R$ between sample and thermal bath of the measurement setup.
\end{abstract}

\section{Introduction}

In magnetic refrigeration cycles, in order to reach the required temperature differences, it is indispensable to run through a cycle many times at moderate cycling frequencies [1]. If a magnetic refrigerant material that exhibits a first order magneto-structural transition is subject to such a field and/or temperature cycling, hysteresis and rate dependence influence the dynamic behaviour of the magnetocaloric effect up to the point that the cooling efficiency is reduced. Therefore, the dynamic of the magnetocaloric effect is subject of recent studies [2-6]. La-Fe-Si compounds are a promising class of materials for magnetic refrigeration applications since they are produced by conventional powder metallurgical routes, do not contain toxic elements and the transition temperature can be easily tuned by Co substitution and/or hydrogenation. The unsubstituted compounds are known to exhibit a first order magnetostructural transition, while the substitution changes gradually the character of the transition from first to second order. Recently, kinetic effects of the temperature induced transition have been studied for $\mathrm{LaFe}_{11.44} \mathrm{Si}_{1.56}$ [2] and the field induced transition of $\mathrm{LaFe}_{11.7} \mathrm{Si}_{1.3}$ [3] and explained in the framework of thermal activation by using the JohnsonMehl-Avrami (JMA) model [7]. However, it was pointed out by [5] that also non-ideal measurement conditions (e.g. heat flow in the measurement setup) leads to similar rate dependencies.

In the presented paper we analyse the dynamics of the field and temperature induced transition of $\mathrm{Co}$ substituted $\mathrm{La}\left(\mathrm{Fe}_{\mathrm{x}} \mathrm{Co}_{\mathrm{y}} \mathrm{Si}_{1-\mathrm{x}-\mathrm{y}}\right)_{13}$ with $x=0.9$ and low $\mathrm{Co}$ content of $y=0.015$ by calorimetry in magnetic field and magnetisation relaxation measurements. $\mathrm{La}\left(\mathrm{Fe}_{\mathrm{x}} \mathrm{Si}_{1-\mathrm{x}}\right)_{13}$ is a pseudo-binary intermetallic compound with cubic $\mathrm{NaZn}_{13}$-type crystalstructure. The binary $\mathrm{LaFe}_{13}$ does not exist, instead substitution with $\mathrm{Al}$ [8] or $\mathrm{Si}$ [9] is necessary to obtain a stable structure. The compounds with high Fe content $(0.8 \leq x \leq 0.9)$ are of special interest due to their Invar like behavior close to the Curie temperature [9]. A sharp increase of magnetisation with decreasing temperature is observed at $T_{c}$. This behavior was attributed to an itinerant electron metamagnetic transition with a large magnetovolume effect [10]. It was shown by neutron diffraction for $x=0.877$ that the transition goes together with an abrupt change of the lattice constant and so an increase of the Fe bond length, which favors the ferromagnetic state [11]. The magnetic transition is evidenced by the fact that a small applied magnetic field induces a transition from the paramagnetic state to the ferromagnetic state with hysteresis in the magnetization curve.

The physical origin of the transition appears to be of two kinds: a) an itinerant electron metamagnetic transition due to a change of electronic structure of the itinerant $d$ electrons induced by the field and $b$ ) a para- to ferromagnetic transition with volume change. A theoretical phenomenological description of the first was found by Wohlfarth and Rhodes [12] and Shimizu [13] based on the Landau theory of the Stoner model. It was modified to take into account the effect of a finite temperature by considering spin fluctuations by Moriya [14] and Yamada [15]. The latter can be described on the basis of the molecular field approximation considering an exchange interaction that depends on the interatomic distance (model of Bean and Rodbell [16]).

In both cases the model assumes a free energy of the system with two local minima corresponding to the ferro- and paramagnetic state where the energy landscape is changed by a magnetic field and/or temperature. In such a system the energy barrier may be overcome by thermal fluctuations which gives rise to relaxation effects and a dependence of the transition on the rate of the driving force. This thermal activation over an energy barrier is considered for the magnetization process in general $[17,18,19]$ and for phase transformations in the framework of the JMA model $[20,21]$. Thermally activated processes consist in the possibility that a small correlated region of volume $v_{0}$ and mass $m_{0}$ makes the transition with the help of the thermal energy $k_{B} T$. Such approach is justified for all transitions of exponential type (e.g. nucleation type) where an energy barrier has to be overcome. This can be a domain wall, for magnetisation processes, or an interface between two phases, for nucleation type phase transitions, that has to overcome a pinning site. The literature has considered single barriers as well as distributions of energy barriers. The average time for the spontaneous overcoming of an energy barrier 
can be reformulated as a problem of first passage for a stochastic process [22]. The result is that when $k_{B} T \ll E_{b}$ the rate $\omega$ at which the activation event occurs, is the Arrhenius law:

$\omega \propto \frac{1}{\tau_{0}} e^{-\frac{E_{b}}{k_{B} T}}$

where $\tau_{0}$ is a microscopic attempt time (typically around $10^{-10}-10^{-13} \mathrm{~s}$ [20] for single domain particles, $10^{-9}-10^{-11} \mathrm{~s}$ for nanocrystalline magnetic materials [22] and $10^{-18}$ $10^{-21} \mathrm{~s}$ for structural transitions in the JMA model [23]), $E_{b}$ is the energy barrier, and $k_{B}$ is the Boltzmann constant.

For magnetocaloric compounds dynamic effects were investigated in the framework of thermal activation for $\mathrm{LaFe}_{11.44} \mathrm{Si}_{1.56}, \mathrm{LaFe}_{11.7} \mathrm{Si}_{1.3}$ and $\mathrm{Gd}_{5} \mathrm{Si}_{2} \mathrm{Ge}_{2}$ [2-4], where typical time constants were derived from relaxation measurements of magnetisation at constant field or susceptibility measurements at different cooling rates. In the present work it is shown that similar rate dependent hysteresis of the transition as observed in [2-4] can be attributed to bad thermal contacts between sample and bath. These effects have to be eliminated as far as possible before studying kinetic effects of the transition.

\section{Experimental}

It was claimed in [5] that rate dependent hysteresis is also due to non-ideal measurement conditions or even heat diffusion effects in the measured sample itself, called 'extrinsic' hysteresis in contrast to the 'intrinsic' one of the magnetic transition itself. The aim of the present work was to separate these two contributions and analyse the origin of the extrinsic hysteresis. Calorimetric and relaxation experiments were performed on Co substituted $\mathrm{La}\left(\mathrm{Fe}_{\mathrm{x}} \mathrm{Co}_{\mathrm{y}} \mathrm{Si}_{1-\mathrm{x}-\mathrm{y}}\right)_{13}$ with $x=0.9$ and low Co content of $y=0.015$. This compound exhibits a first order magnetostructural transition with the transition temperature slightly shifted towards higher temperatures with respect to the unsubstituted $\mathrm{La}\left(\mathrm{Fe}_{0.9} \mathrm{Si}_{0.1}\right)_{13}$. The sample was prepared by Vacuumschmelze $\mathrm{GmbH}$ and Company KG by powder metallurgy [24].

Calorimetric measurements were performed by a home-built low temperature calorimeter, similar to [25]. With this setup it is possible to measure: a) the isothermal entropy change $\left.\Delta s(H)\right|_{T}$ obtained by applying a magnetic field (see figure 1) and b) the specific heat $c_{p}(T)$ as a function of temperature in isofield from which $\left.s(T)\right|_{H^{-} S_{0}}$ is derived (see figure 2). The temperature can be changed in the range from $77-300 \mathrm{~K}$. The measurement is based on the evaluation of heat flux from the sample to a thermal bath measured by Peltier cells. The principle of the setup follows the one of a differential scanning calorimeter with two Peltier cells connected differentially. One cell is empty and compensates for heat flux losses towards a thermal screen. The sample is placed on the other cell. The cells are thin film Peltier cells (Micropelt MPG D602) with very good dynamic behavior due to the reduced thickness (heat diffusion time constant $<0.5 \mathrm{~s}$ ). The size of the cells is $2.5 \times 2.5 \times 1.07 \mathrm{~mm}^{3}$. The setup is placed in an Oxford MicrostatHe cryostat, whose cold finger constitutes the thermal bath. The cryostat works with liquid nitrogen as the refrigerating fluid and the temperature is changed by an electric heater controlled by the Oxford digital controller. The magnetic field is applied by an electromagnet $\left(H_{\max }=1.8 \mathrm{~T}\right)$.

For magnetisation relaxation measurements an alternating gradient force magnetometer (AGFM) was employed.

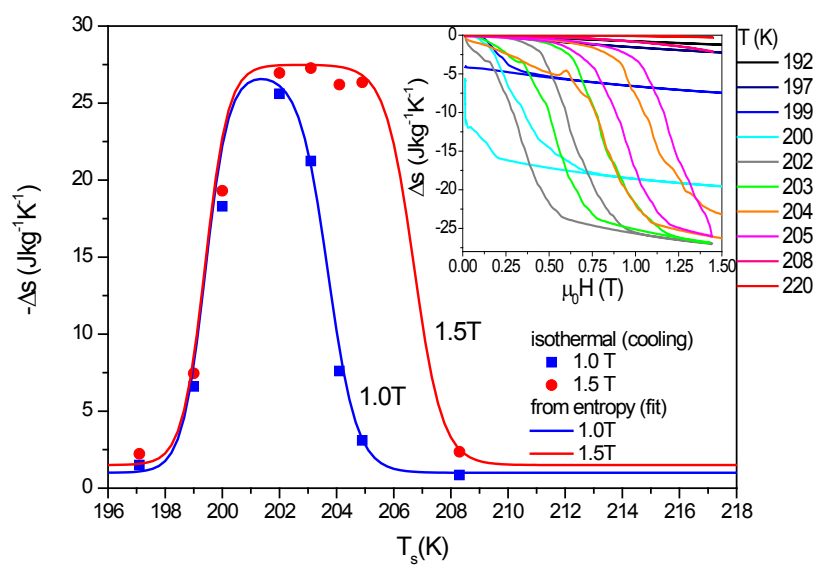

Fig. 1. $\left.\Delta s(H)\right|_{T}$ measurement at various temperatures around the first order transition. The solid lines are obtained from a fit of entropy measurements (see figure 2).

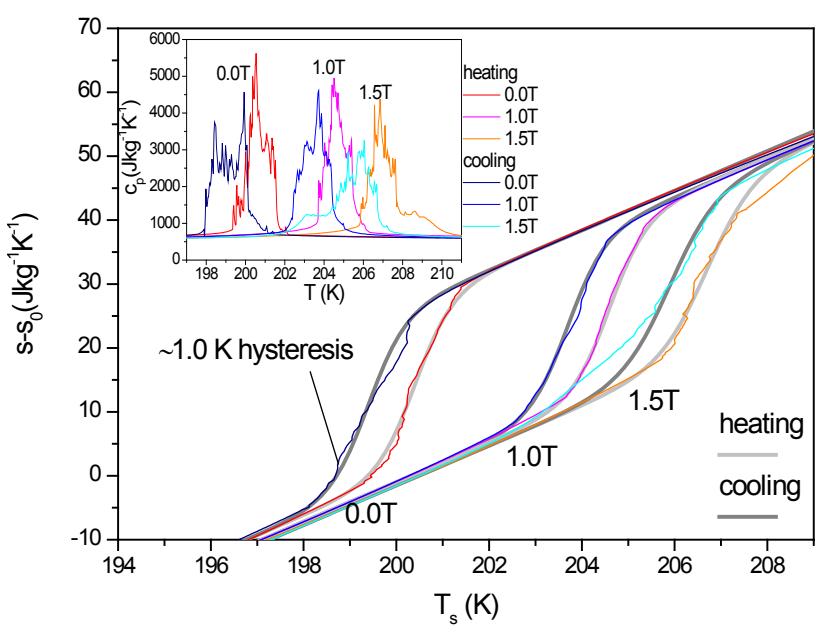

Fig. 2. $\left.s(T)\right|_{H^{-} S_{0}}$ obtained from $c_{p}(T)$ measurements (inset) at various magnetic fields. The curves were corrected for the dynamic of the Peltier cell and the thermal contact resistance between sample and cell as described in the text.

\section{Results and discussion}

For both $\left.\Delta s(H)\right|_{T}$ and $c_{p}(T)$ measurements a strong rate dependence is observed. In order to measure the dynamics of a phase transition it is necessary to consider the dynamic of the measurement setup itself. In the case of the presented calorimeter, the Peltier cell itself has a finite heat capacity and heat conductivity and therefore the heat diffusion through the cell occurs with a typical time constant $\tau$. A correction of the measurements has to be performed as described in [25]. This correction is rather small for the micro Peltier cells $(\tau=0.5 \mathrm{~s})$ used in the present setup. However, even after the correction a rate dependence remains as shown in figure 3 and 4 . For many calorimeters the rate dependent $c_{p}(T)$ measurement is a known problem and attributed to the thermal contact 
resistance between heat sensor and sample [26]. In an ideal first order transition the sample temperature $T_{s}$ remains constant. Thus, a temperature lag

$T_{b}-T_{s}=R q$

between bath temperature $T_{b}$ (measured) and sample temperature $T_{s}$ exists. This lag is proportional to the heat flux $q$ with the thermal contact resistance $R$ between bath and sample as proportionality constant. This resistance depends mainly on the surface roughness of sample and cell and on the contact medium used (setup in vacuum/Ar gas, thermal grease/varnishes, etc.). It leads to a measured $c_{p}{ }^{*}\left(T_{b}\right)$ that increases linearly with a slope proportional to the temperature rate $\dot{T}_{b}$ and the contact resistance $R$ :

$c_{p}^{*}\left(T_{b}\right)=\frac{\left(T_{b}-T_{t}\right)}{R \dot{T}_{b} m}$

( $m$ is the mass of the sample and $T_{t}$ the transition temperature). A linear fit of the measured curves gives the transition temperature and an approximate value for the thermal contact resistance (see figure 3). Two different contact media (silver varnish and thermal grease Apiezon $\mathrm{N}$ ) were used to show the difference in contact resistance. The obtained value can be used to plot the heat capacity as a function of the sample temperature $T_{s}$ which eliminates the temperature rate dependence and allows to evaluate the transition hysteresis (as shown in figure 2).

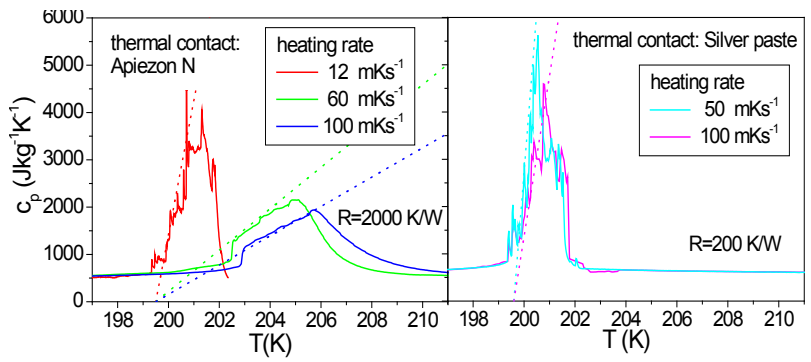

Fig. 3. $c_{p}(T)$ measurements at various temperature rates using different thermal contact media between sample and cell. The dotted lines are fits according to equation (3) using a thermal contact resistance $R$.

Figure 4 shows $\left.\Delta s(H)\right|_{T}$ at various field rates using two different contact media. A rate dependent hysteresis is observed which is strongly influenced by the choice of the contact medium. In this measurement $T_{b}$ remains constant but due to the magnetocaloric effect the sample temperature changes. By a reasoning similar to the temperature lag one, we can define an equivalent magnetic field [26]

$H_{e q}-H=\frac{d H}{d T_{s}} R q$

which allows to correct the measurement for the thermal contact resistance (see figure 5). The contact resistance is a fitting parameter chosen such to make the curves measured at different rates coincide [28]. Using this method, all dynamic effects are eliminated. A reasonable conclusion is therefore, that all dynamic can be attributed to the thermal contact resistance between sample and cell. If there are residual dynamic effects of the transition in
$\mathrm{LaFe}_{11.7} \mathrm{Co}_{0.195} \mathrm{Si}_{1.105}$, that can be attributed to thermal activation, they might be too small to be observed in calorimetric measurements.

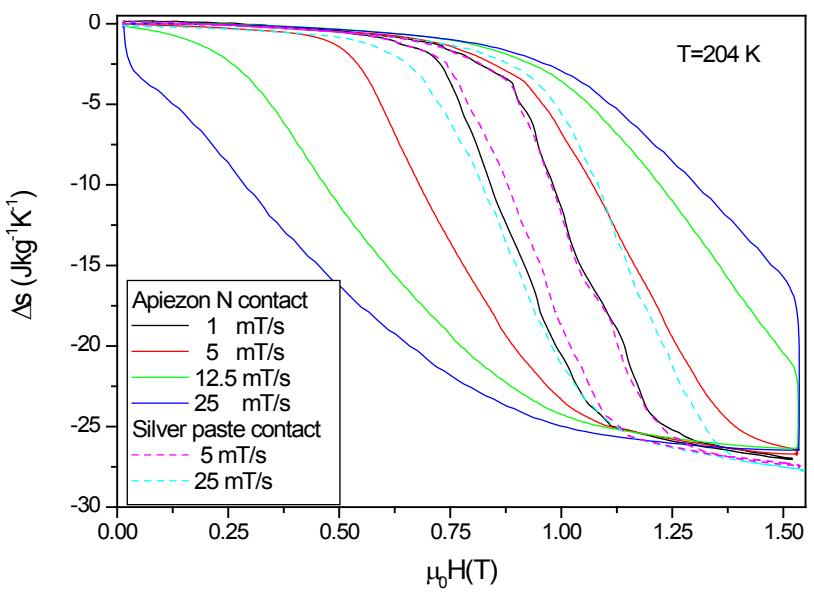

Fig. 4. $\left.\Delta s(H)\right|_{T}$ measurements at various field rates using different thermal contact media between sample and cell.

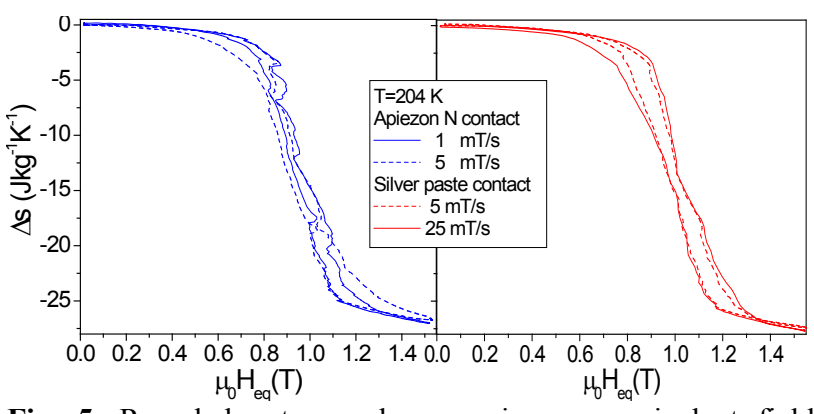

Fig. 5. Rescaled entropy change using an equivalent field according to equation (4).

In order to test this hypothesis or to verify effects of thermal activation of the transition in $\mathrm{LaFe}_{11.7} \mathrm{Co}_{0.195} \mathrm{Si}_{1.105}$ magnetisation relaxation measurements were performed by AGFM (see figure 6). At a fixed temperature close to the transition temperature the field was driven with a constant rate up to a certain field and then kept constant for a waiting time of a few minutes. The measurement conditions are similar to the ones in the $\left.\Delta s(H)\right|_{T}$ measurement, i.e. bath temperature is kept constant but sample temperature varies during the transition. Therefore, also in this case, a correction for an equivalent field has to be performed. Instead of the heat flux $q=T \frac{d S}{d t}$ the measured quantity here is the magnetisation $M$, therefore equation (4) has to be replaced by

$H_{e q}-H \approx \frac{d H}{d T_{S}} R T_{b} \frac{\Delta S}{\Delta M} \frac{d M}{d t}$

where $\frac{\Delta S}{\Delta M}=-\frac{1}{\mu_{0}} \frac{d H}{d T_{S}}$ (Clausius-Clapeyron equation). The inset (b) of figure 6 shows the rescaled magnetisation curves. Again it is possible by using a thermal contact resistance of $4000 \mathrm{~K} / \mathrm{W}$ to eliminate all dynamic effect and the measured relaxation curves coincide with the major hysteresis curve.

Since the determination of the thermal contact resistance is based only on fitting (an exact determination using the thermal conductivity values of the contact medium is rather difficult since thickness of the medium 
layer and the surface roughnesses are not exactly known, in addition voids that form during evacuation of the setup and non-uniformities of the contact complicate further the calculation [28]), remains the doubt that the dynamic of the transition itself is obscured by the correction procedure. This would significate that any increase of hysteresis of the transition entropy, e.g. due to thermal activation, is eliminated by the rescaling of the curves. However, the strong influence of the thermal contact resistance on the rate dependent hysteresis is clearly shown in figure 4, using two different contact media. Since all kind of measurements, calorimetric as well as magnetisation, present this problem, an analysis of the effect of the contact resistance has to be performed before applying models, as thermal activation, to the experimentally observed kinetics. As an example, we discuss the data presented in Fig.1 of [3]: The observed difference in field hysteresis after allowing a relaxation time of $t_{w}^{e}=1 \mathrm{~s}$ and $1600 \mathrm{~s}$ is $\sim 0.1 \mathrm{~T}$. By using equation (5) and the values $\left.\mu_{0} \frac{d M}{d t}\right|_{\text {slow }}=1.7 \cdot 10^{-3} \mathrm{~T} / \mathrm{s}$ for $t_{w}^{e}=1600 \mathrm{~s}$ and $\left.\mu_{0} \frac{d M}{d t}\right|_{\text {fast }}=3.2 \cdot 10^{-2} \mathrm{~T} / \mathrm{s}$ for $t_{w}^{e}=1 \mathrm{~s}$, a thermal contact resistance of $R \sim 1900 \mathrm{~K} / \mathrm{W}$ is calculated. This value, which is very similar to the ones obtained in our experiments, allows to eliminate all rate dependent effects.

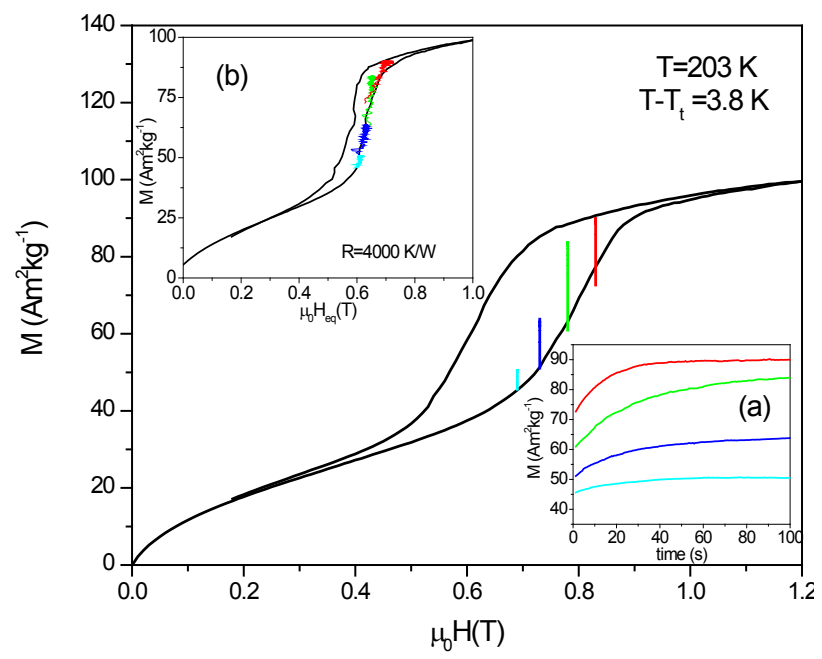

Fig. 6. Magnetisation relaxation measurements at $203 \mathrm{~K}$. Inset (b) shows the rescaled curve using an equivalent field according to equation (5).

\section{Conclusions}

The observed rate dependence of the magnetocaloric effect and increase in hysteresis of a first order transition with the rate of the driving force are ascribed mainly to heat diffusion effects from sample to the measurement setup and can be described by a thermal contact resistance $R$. Therefore, in order to study experimentally the dynamic behaviour of a first order transition, it is necessary to make a detailed analysis of the heat diffusion in the employed measurement setup. Such an analysis is indispensable for calorimetric as well as for magnetisation measurements. By correcting the measurements for the effect of the thermal resistance the rate dependence can be eliminated concluding that effects of thermal activation of the first order transition in the Co substituted La-Fe-Si compound $\mathrm{LaFe}_{11.7} \mathrm{Co}_{0.195} \mathrm{Si}_{1.105}$ are negligible.

\section{References}

1. M. Kuzmin, APL, 90, 251916 (2007)

2. A. Fujita and H. Yako, J. All. Comp., in Press

3. H. Zhang, F. Wang, T. Zhao, S. Zhang, J. Sun and B. Shen, PRB, 70, 212402 (2004)

4. J. Leib, J.E. Snyder, T.A. Lograsso, D.Schlagel and D.C. Jiles, JAP, 95, 6915 (2004)

5. J.D. Moore, K. Morrison, K.G. Sandeman, M. Katter and L.F. Cohen, APL, 95, 252504 (2009)

6. N.T. Trung, J.C.P. Klaasse, O. Tegus, D.T. Cam Thanh, K.H.J. Buschow and E. Brueck, J.Phys.D :Appl.Phys., 43, 015002 (2010)

7. M. Avrami, J. Chem. Phys., 7, 1103 (1939)

8. R.B. Helmholdt, T.T.M. Palstra, J.A. Mydosh, G.J. Nieuwenhuys, A.M. van der Kraan, and K.H.J. Buschow, PRB, 34, 169 (1985)

9. T.T.M. Palstra, J.A. Mydosh, G.J. Nieuwenhuys, A.M. van der Kraan, and K.H.J. Buschow, JMMM, 36, 290 (1983)

10. A. Fujita, Y. Akamatsu, and K. Fukamichi, JAP, 85, 4756 (1999)

11. F. Wang, G. Wang, F. Hu, A. Kurbakov, B. Shen, and Z. Cheng, J.Phys: Cond. Mat., 15, 5269 (2003)

12. E.P. Wohlfarth and P. Rhodes, Phil. Mag., 7, 1817 (1962)

13. M. Shimizu, J. Phys., 43, 155 (1982)

14. T. Moriya, Spin Fluctuations in Itinerant-Electron Magnetism, Springer-Verlag, Berlin, 1985

15. H. Yamada, PRB, 47, 11211 (1993)

16. C.P. Bean and D.S. Rodbell, Phys. Rev., 126, 104 (1962)

17. R. Street and J. Wolley, Proc. Phys. Soc., London, Sect. A 62, 562 (1949)

18. S. Morup and E. Tronc, PRL, 72, 3278 (1994)

19. V. Basso, C. Beatrice, M. LoBue, P. Tiberto, and G. Bertotti, PRB, 61, 1278 (2000).

20. X.P. Wang, G. Corbel, S. Kodjikian, Q.F. Fang and P. Lacorre, Sol. Stat. Chem., 179, 3338 (2006)

21. F. Liu, S.J. Song, F. Sommer and E.J. Mittemeijer, , Acta Mat., 57, 6176 (2009)

22. C. W. Gardiner, Handbook of Stochastic Methods, Springer-Verlag, Berlin (1985)

23. F. Liu, F. Sommer, C. Bos, and E.J. Mittemeijer, Int. Mat. Rev., 52 (4), 193 (2007)

24. M. Katter, V. Zellmann, G.W. Reppel and K. Uestuener, IEEE Trans. Mag., 44 (11), 3044, (2008)

25. V. Basso, C.P. Sasso and M.Kuepferling, Rev. Sci. Instr., 81, 113904 (2010)

26. T. Plackowski, Y. Wang and A. Junod, Rev. Sci. Instr., 73 (7), 2755, (2002)

27. V. Basso, C.P. Sasso, M. Lo Bue, J. Magn. Magn. Mater. 316, 262, (2007)

28. V. Basso, C.P. Sasso and M. Kuepferling, Proc. Thermag V, Grenoble (to be published) 